\title{
El enfoque de aprendizaje en alumnos de máster: Análisis del cuestionario R-SPQ-2F en tres cursos académicos
}

\author{
Leiva-Brondo, M. ${ }^{\text {a }}$, Cebolla-Cornejo, J. ${ }^{\text {b }}$, Peiró, R. ${ }^{\text {c }}$, Pérez-de-Castro, A. ${ }^{d}$ \\ amileibro@btc.upv.es, Departamento de Biotecnología bjaicecor@btc.upv.es, Departamento de \\ Biotecnología, ${ }^{2}$ ropeibar@btc.upv.es, Departamento de Biotecnología, ${ }^{\mathrm{d}}$ anpede1@btc.upv.es, Departamento \\ de Biotecnología, Universitat Politècnica de València.
}

\begin{abstract}
Students' approach to learning is variable depending on the student's personal and situational factors. Although there are different classifications, deep and superficial approaches appear as the most common. In this study, the R-SPQ-2F questionnaire has been used in students of a second master's year for three consecutive academic courses. The result showed that students present a greater deep approach than superficial and the questionnaire is valid in the educational context conducted. The gender of the students was not a differential factor, but the course analyzed indicated that there was variability among participating students between courses. The intrinsic motivation of the students and the methodology used in the subject seemed the most influential factors in promoting the deep learning of the students.
\end{abstract}

Keywords: Deep and Surface approach to learning, learning context, active teaching methodologies

\section{Resumen}

El enfoque de aprendizaje de los estudiantes es variable dependiendo de factores personales y situacionales. Aunque hay distintas clasificaciones, los enfoques profundo y superficial aparecen como los más comunes. En el presente estudio se ha utilizado el cuestionario $R-S P Q-2 F$ en alumnos de una asignatura de segundo curso de máster durante tres cursos académicos consecutivos. El resultado mostró que los alumnos manifiestan un mayor enfoque profundo que superficial y el cuestionario es valido en el contexto educativo realizado. El género de los estudiantes no fue un factor diferencial, pero si el curso analizado, indciando que habia variabilidad entre los estudiantes participantes entre cursos. La motivación intrínseca de los estudiantes y la metodología usada en la asignatura parecían los factores más influyentes en favorecer el aprendizaje profundo de los estudiantes.

Palabras clave: Enfoque profundo y superficial de aprendizaje, contexto de aprendizaje, metodologías activas de enseñanza

\section{Introducción}

El enfoque de aprendizaje de los alumnos es uno de los factores más determinantes en el rendimiento de los alumnos y en la consecución de los objetivos de aprendizaje de una asignatura (Marton and Säljö, 1976b, 1976a; Biggs, 1987). Aunque el enfoque de aprendizaje puede clasificarse de distintas formas, existen dos grandes escalas: aprendizaje profundo y aprendizaje superficial (Marton and Säljö, 1976b; Entwistle and Entwistle, 2003). El aprendizaje profundo se caracteriza por una mayor reflexión y 
comprensión y supone que el alumno acepta el aprendizaje como una responsabilidad intrínseca, mientras el aprendizaje superficial se caracteriza por una falta de reflexión y un esfuerzo dedicado a superar la asignatura con el mínimo esfuerzo (Justicia et al., 2008; Fryer et al., 2012; Frăsineanu, 2013).

El enfoque de aprendizaje no es una característica fija del alumno y varía en función de factores personales y situacionales (Biggs, 1987). Entre los factores personales se puede incluir la personalidad, pero también la edad, el género o los condicionantes socioculturales (Gijbels et al., 2005; Salamonson et al., 2013). Por ejemplo, se ha detectado que en general que los estudiantes más jóvenes suelen tener un enfoque más superficial (Gow and Kember, 1990; Richardson and King, 1998; Gijbels et al., 2005; Richardson, 2013). También se han observado diferencias entre países y contextos culturales (Biggs, Kember and Leung, 2001; Justicia et al., 2008; Immekus and Imbrie, 2010). Factores situacionales como el tipo de estudio, la asignatura, las actividades en el aula o el sistema de evaluación modifican el enfoque del aprendizaje del alumno, que lo adapta a cada contexto (Biggs and Tang, 2007; Rubin et al., 2018). Se ha detectado que el enfoque suele ser más profundo en primeros cursos universitarios que en cursos finales, indicando que el ambiente formativo no requiere una implicación del alumno en su formación y que se fomenta un aprendizaje más estratégico enfocado en el sistema de evaluación (Biggs, 1987; Zeegers, 2001).

Se han desarrollado diversos instrumentos para evaluar el enfoque de aprendizaje de los estudiantes tales como: Study Attitudes and Methods Revised Short Form (SAMS Short Form) (Michael, Michael and Zimmerman, 1985), Revised Approaches to Studying Inventory (RASI) (Entwistle and Tait, 1995) modificado a Approaches and Study Skills Inventory for Students (ASSIST) (Tait, Entwistle and McCune, 1998; Entwistle and Hilary, 2013), Inventory of Learning Process-Revised (ILP-R) (Schmeck, Ribich and Ramanaiah, 1977), Approaches to Learning and Studying Inventory (ALSI) (Entwistle, McCune and Hounsell, 2002), Learning and Study Inventory Strategies (LASSI) (Weinstein, 1987), or Inventory of Learning Styles (ILS) (Vermunt, 1994). Pero uno de los más utilizados es el Study Process Questionnaire (SPQ) (Biggs, 1987), que fue posteriormente revisado (R-SPQ-2F) (Biggs, Kember and Leung, 2001), y ha sido validado en diversos estudios y condiciones (Justicia et al., 2008; Immekus and Imbrie, 2010; Socha and Sigler, 2014).

El cuestionario R-SPQ-2F consiste en 20 preguntas con una escala Likert de 5 puntos (Biggs, Kember and Leung, 2001) y pretende estimar el comportamiento de los alumnos en un contexto específico de aprendizaje donde el alumno opta por un enfoque de aprendizaje en función de factores intrísecos y extrínsecos. El cuestionario tiene dos escalas principales: profundo (DA) y superficial (SA) y dos subescalas: estratégica (DS y SS) y motivacional (DM y SM), aunque estas dos subescales no se han encontrado consistentes en diversos estudios (Justicia et al., 2008; Immekus and Imbrie, 2010; Socha and Sigler, 2014).

Los resultados del cuestionario R-SPQ-2F obtenidos por Biggs y colaboradores en Hong Kong (Biggs, Kember and Leung, 2001) han sido comparados con los obtenidos en distintos países occidentales (España (Justicia et al., 2008), Japón (Fryer et al., 2012), Estados Unidos (Immekus and Imbrie, 2010; Socha and Sigler, 2014), Países Bajos (Stes, de Maeyer and Van Petegem, 2013) o Noruega (Zakariya, 2019)) y en otros países con contextos culturales más distintos (Ghana, (Mogre and Amalba, 2014), Malasia (Swee Choo Goh, 2006) o Arabia Saudi (Shaik et al., 2017) obteniendo resultados varabibles dependientes del context educativo y otros factores.

En estudios relacionados con las ciencias de la vida se han hecho algunas aproximaciones (Leiva-Brondo et al., 2018a, 2018b, 2019), sin embargo es necesario realizar dichos estudios en diversos años para ver la posible variación entre cursos, con objeto de evaluar la consistencia y obtener información que permita determinar las mejores estrategias de enseñanza que fomenten el aprendizaje profundo de los alumnos. En 
el presente estudio se analiza el enfoque de aprendizaje de estudiantes de segundo curso de máster durante tres cursos consecutivos y se analiza la implicación de los resultados.

\section{Objetivos}

En el presente estudio se pretende evaluar la fiabilidad del cuestionario R-SPQ-2F en el contexto de una asignatura de máster relacionada con las ciencias de la vida y analizar los valores y la relación entre las distintas escalas del cuestionario con el objetivo de determinar las mejores estrategias de aprendizaje en este contexto. Se analizan las diferencias en las escalas en función del curso y el género del alumno así como las correlaciones existentes y la fiabilidad interna del cuestionario.

\section{Desarrollo de la innovación}

Los alumnos participantes en el estudio pertenecían a la titulación de máster en Mejora Genética Vegetal que se imparte en la Universitat Politècnica de València (UPV). Los estudiantes cursaban la asignatura Mejora Genética del Rendimiento en los Sistemas Agrarios de segundo curso con una carga lectiva de 5 ECTS (European credit transfer system, 50 horas lectivas) organizados en 4 ECTS de teoría de aula y 1 ECTS de prácticas de informática. El estudio se realizó durante tres cursos académicos consecutivos (2017-18, 2018-19 y 2019-20) con 13, 17 y 18 alumnos matriculados respectivamente (29 mujeres y 19 hombres).

El cuestionario R-SPQ-2F se les proporcionó a los alumnos a través de la plataforma de aprendizaje PoliformaT (basada en Sakai) al inicio de la asignatura y se les informó de la finalidad del estudios. El estudio fue voluntario y no tenía relación con ningún acto de evaluación de la asignatura. La versión del cuestionario utilizada fue una traducción realizada por Muñoz San Roque y colaboradores (Muñoz San Roque, Prieto Navarro and Torre Puente, 2012). Se utilizaron los programas Microsoft $\subset$ Excel y Statgraphics centurión XVII (Statpoint Technologies, Inc.) y se calcularon los valores medios de las diferentes aproximaciones, las correlaciones entre éstos y los valores alfa de Cronbach de fiabilidad del cuestionario.

\section{Resultados y discusión}

La tasa de respuesta de los estudiantes fue alta (media $=85,4 \%$ ) durante los tres cursos académicos evaluados (Tabla 1). En las escalas principales, los valores de enfoque profundo (DA) fueron superiores a los valores de enfoque superficial (SA) en todos los cursos y la diferencia superior a cero con solo 4 casos de estudiantes con valores SA superiores a DA (datos no mostrados). En general estos resultados concuerdan con los obtenidos en otros estudios donde los alumnos muestran un mayor enfoque profundo que superficial (Leung, Ginns and Kember, 2008; Emilia, Bloomfield and Rotem, 2012; Mogre and Amalba, 2014; Jeong et al., 2017). Los alumnos objeto de estudio pertenecen a una titulación de máster y se ha visto que la edad es un factor que influye en el enfoque de aprendizaje, mostrando los alumnos mayores enfoque más profundo que superficial (Zeegers, 2001; Richardson, 2013; Rubin et al., 2018). Además, los estudios de máster suelen tener una elección más intríseca que los estudios de grado, por lo que el alumno está más motivado para su aprendizaje y menos motivado por la calificación que obtenga en la asignatura. Para los valores de DA no se encontraron diferencias significativas entre cursos, pero si para SA, con un valor superior en el último curso respecto al primero, y consecuentemente menores diferencias entre DA y SA. Para las escalas secundarias los valores de DM y DS fueron superiores a los 
valores de SS y SM en todos los cursos, y solo se encontraron diferencias entre cursos en los valores de SM. El entorno de aprendizaje es un factor que influye en el enfoque de aprendizaje del estudiante (Biggs and Tang, 2007; Rubin et al., 2018). La metodología utilizada en los tres cursos ha sido similar y no ha habido cambios en el profesorado, por lo que las diferencias pueden deberse a factores personales de los estudiantes de cada curso y sus expectativas respecto a la asignatura. El genero no fue un factor significativo en ninguna de las escalas. Estudios realizados tampoco encuentran diferencias relacionadas con el género en el enfoque de aprendizaje del estudiante, aunque en algunos casos sí ligado a la edad (Richardson, 2013; Jeong et al., 2017). La distribución de los alumnos en función de los valores obtenidos en DA y SA (Figura 1) muestra que igual número de alumnos mostraron valores superiores al DA medio e inferiores al SA medio que alumnos con valores opuestos (DA inferior a la media y SA superior a la media) y menos alumnos con enfoques intermedios al igual que en otros estudios (Jeong et al., 2017). Esto que puede indicar que los alumnos se implican en la asignatura con un enfoque profundo o no se implican mostrando un enfoque superficial, pero no son tan frecuentes enfoques intermedios.

Tabla 1. Número de alumnos que respondieron el cuestionario por curso y género de los alumnos (\%) y media y error estándar de las escalas de cuestionario $R-S P Q-2 F$ en la aproximación profunda (DA), aproximación superficial (SA), diferencia entre DA y SA,hipótesis nula, motivación profunda (DM), estrategia profunda (DS), motivación superficial (SM) y estrategia superficial (SS).

\begin{tabular}{|c|c|c|c|c|c|c|c|}
\hline & $\begin{array}{l}\mathrm{N}^{0} \text { respuestas } \\
(\% \text { alumnos } \\
\text { matriculados) }\end{array}$ & DA & & $\mathbf{S A}$ & & Diferencia DA-SA & $\begin{array}{c}\text { Hipótesis nula } \\
\text { DA-SA }\end{array}$ \\
\hline \multicolumn{8}{|l|}{ Curso } \\
\hline 2017-18 & $11(0,85)$ & $3,20 \pm 0,20$ & $\mathrm{a}$ & $1,77 \pm 0,14$ & $\mathrm{a}$ & $1,43 \pm 0,28$ & $* * *$ \\
\hline 2018-19 & $15(0,88)$ & $3,15 \pm 0,14$ & $\mathrm{a}$ & $2,00 \pm 0,13$ & $\mathrm{ab}$ & $1,15 \pm 0,24$ & $* * *$ \\
\hline $2019-20$ & $15(0,83)$ & $2,86 \pm 0,16$ & $\mathrm{a}$ & $2,31 \pm 0,15$ & $\mathrm{~b}$ & $0,55 \pm 0,17$ & $* *$ \\
\hline \multicolumn{8}{|l|}{ Género } \\
\hline Femenino & $24(0,83)$ & $3,08 \pm 0,12$ & $\mathrm{a}$ & $1,99 \pm 0,11$ & $\mathrm{a}$ & $1,09 \pm 0,19$ & $* * *$ \\
\hline Masculino & $17(0,89)$ & $3,03 \pm 0,16$ & $\mathrm{a}$ & $2,14 \pm 0,14$ & $\mathrm{a}$ & $0,89 \pm 0,20$ & $* * *$ \\
\hline Total & $41(0,85)$ & $3,06 \pm 0,09$ & & $2,05 \pm 0,09$ & & $1,01 \pm 0,14$ & $* * *$ \\
\hline
\end{tabular}

\begin{tabular}{ccccccccc}
\hline & DM & $\mathbf{x}$ & $\mathbf{D S}$ & $\mathbf{x}$ & $\mathbf{S M}$ & $\mathbf{x}$ & $\mathbf{S S}$ & $\mathbf{x}$ \\
\hline Curso & & & & & & & & \\
$2017-18$ & $3,22 \pm 0,22$ & $\mathrm{a}$ & $3,18 \pm 0,19$ & $\mathrm{a}$ & $1,55 \pm 0,14$ & $\mathrm{a}$ & $2,00 \pm 0,20$ & $\mathrm{a}$ \\
$2018-19$ & $3,27 \pm 0,16$ & $\mathrm{a}$ & $3,04 \pm 0,16$ & $\mathrm{a}$ & $1,63 \pm 0,11$ & $\mathrm{a}$ & $2,37 \pm 0,18$ & $\mathrm{a}$ \\
$2019-20$ & $3,01 \pm 0,17$ & $\mathrm{a}$ & $2,71 \pm 0,21$ & $\mathrm{a}$ & $2,13 \pm 0,16$ & $\mathrm{~b}$ & $2,48 \pm 0,16$ & $\mathrm{a}$ \\
\hline Género & & & & & & & & \\
Femenino & $3,20 \pm 0,13$ & $\mathrm{a}$ & $2,96 \pm 0,14$ & $\mathrm{a}$ & $1,67 \pm 0,11$ & $\mathrm{a}$ & $2,32 \pm 0,14$ & $\mathrm{a}$ \\
Masculino & $3,11 \pm 0,17$ & $\mathrm{a}$ & $2,95 \pm 0,18$ & $\mathrm{a}$ & $1,96 \pm 0,14$ & $\mathrm{a}$ & $2,31 \pm 0,17$ & $\mathrm{a}$ \\
\hline & & & & & & & & \\
\hline Total & $3,16 \pm 0,10$ & & $2,96 \pm 0,11$ & & & & & \\
\hline
\end{tabular}




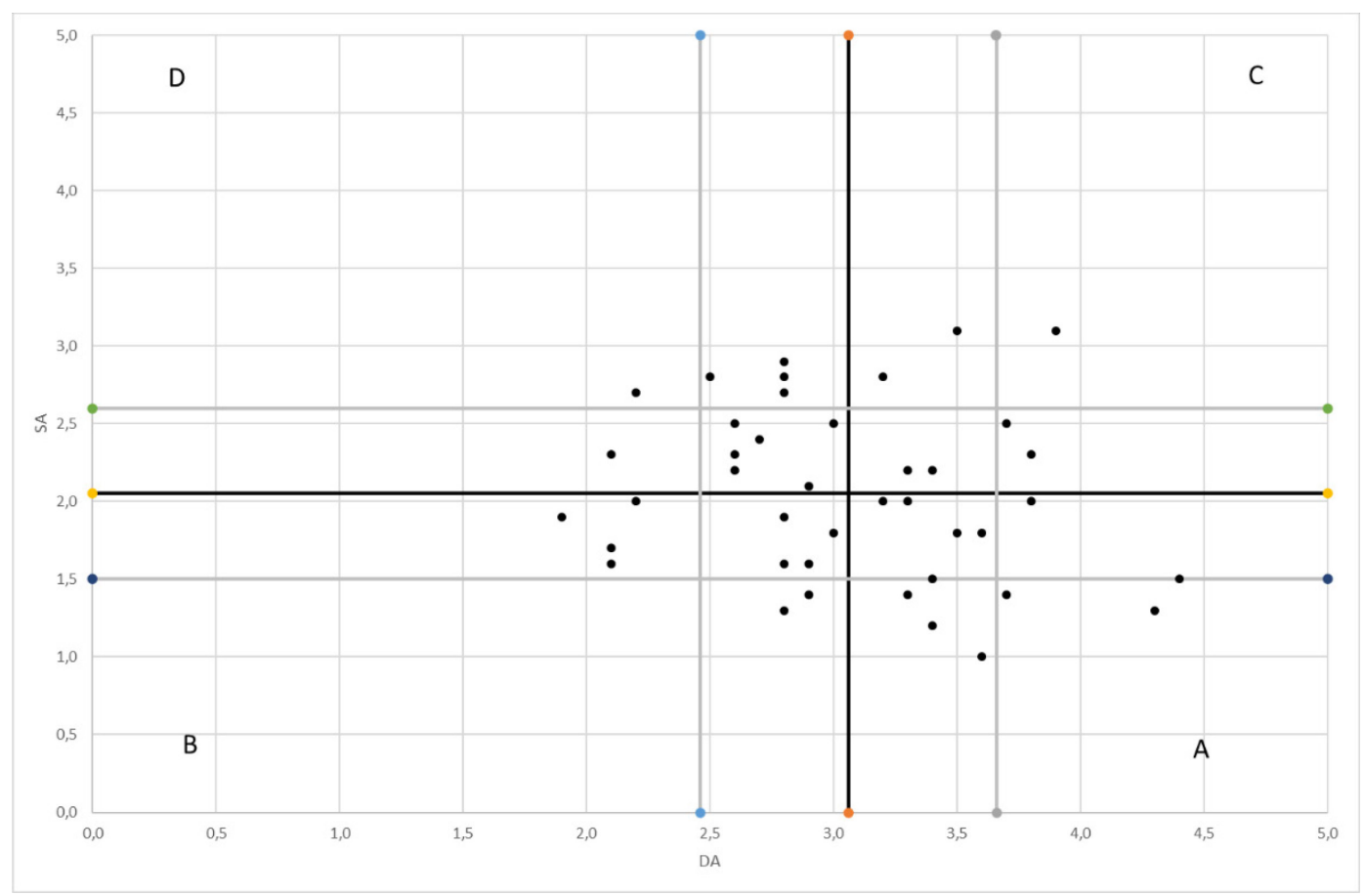

Figura 1. Distribución de los valores para la aproximación profunda (DA) y aproximación superficial (SA) al aprendizaje de cada estudiante. La líneas Negras muestra en valore medio para DA y SA y las líneas grises la media más o menos la desviación estándar.Las letras A, B, C y D identifican los cuadrantes del gráfico

Las correlaciones entre las distintas escalas y subescalas se muestran en la tabla 2, indicando altas correlaciones positivas entre las escalas principales y las subescalas secundarias relacionadas, si bien no se hayan correlaciones significativas entre DA y SA ni entre las subscalas. Estos resultados han sido similares a los de otros estudios (Immekus and Imbrie, 2010; Socha and Sigler, 2014) y coinciden con los esperados por Biggs y colaboradores (Biggs, Kember and Leung, 2001), lo parece confirmar que las dos escalas principales son más consistentes que las subescalas. El desarrollo de nuevos cuestionarios puede permitir la localización de nuevas escalas como el enfoque estratégico que pretende medir una de las subescalas del cuestionario. Un ejemplo es el cuestionario desarrollado por Entwistle y colaboradores (Tait, Entwistle and McCune, 1998; Entwistle and Hilary, 2013), donde se distinguen tres escalas de enfoque de aprendizaje (profundo, superficial y estratégico).

Tabla 2. Correlación entre los distintas aproximaciones de aprendizaje de las escalas de cuestionario R-SPQ-2F de los 41 cuestionarios. Aproximación profunda (DA), aproximación superficial (SA), motivación profunda (DM), estrategia profunda (DS), motivación superficial (SM) y estrategia superficial (SS).

\begin{tabular}{cccccc}
\hline & DA & SA & DM & DS & SM \\
\hline SA & $-0,18 \mathrm{NS}$ & & & & \\
DM & $0,87 * * *$ & $-0,10 \mathrm{NS}$ & & & \\
DS & $0,90 * * *$ & $-0,21 \mathrm{NS}$ & $0,57 * * *$ & & \\
SM & $-0,07 \mathrm{NS}$ & $0,87 * * *$ & $-0,04 \mathrm{NS}$ & $-0,09 \mathrm{NS}$ & \\
SS & $-0,23 \mathrm{NS}$ & $0,91 * * *$ & $-0,13 \mathrm{NS}$ & $-0,27 \mathrm{NS}$ & $0,58 * * *$ \\
\hline \multicolumn{7}{c}{$* * *: \mathrm{P}<0,001 ; * *: 0,001<\mathrm{P}<0,01 ; \mathrm{NS} \mathrm{P}>0,05$} \\
\end{tabular}


La fiabilidad interna del cuestionario fue evaluada con el coeficiente alfa de Cronbach con valores superiores a 0,7 para las escalas principales lo que avala el cuestionario (Hundleby and Nunnally, 2006), pero muestra valores inferiores para las subescalas. Diferentes estudios en distintos países han analizado la consistencia del cuestionario y han detectado distintas asociaciones entre las preguntas según el contexto cultural (Justicia et al., 2008; Immekus and Imbrie, 2010; Socha and Sigler, 2014; Zakariya, 2019) y avalan la estructura en dos escalas frente a una estructura en escalas y subescalas. Serían necesarios estudios confirmatorios en nuestro contexto educativo para avalar esas conclusiones, aunque los datos parciales aquí obtenidos así lo parecen indicar.

Tabla 3. Valores del coeficiente alfa de Cronbach (banda inferior de confianza del $95 \%$ ) entre las distintas escalas de cuestionario $R-S P Q-2 F$ de los 41 cuestionarios evaluados. Aproximación profunda (DA), aproximación superficial (SA), motivación profunda (DM), estrategia profunda (DS), motivación superficial (SM) y estrategia superficial (SS).

\begin{tabular}{ccccccc}
\hline & DA Media & SA Media & DM Media & DS Media & SM Media & SS Media \\
\hline Curso & & & & & & \\
$2017-18$ & $0,87(0,85)$ & $0,75(0,70)$ & $0,82(0,79)$ & $0,70(0,64)$ & $0,67(0,60)$ & $0,67(0,60)$ \\
$2018-19$ & $0,73(0,67)$ & $0,69(0,63)$ & $0,58(0,50)$ & $0,62(0,54)$ & $0,52(0,42)$ & $0,55(0,46)$ \\
$2019-20$ & $0,80(0,72)$ & $0,73(0,61)$ & $0,68(0,52)$ & $0,80(0,71)$ & $0,52(0,29)$ & $0,54(0,32)$ \\
\hline Género & & & & & & \\
Femenino & $0,79(0,70)$ & $0,77(0,68)$ & $0,67(0,52)$ & $0,73(0,60)$ & $0,68(0,52)$ & $0,62(0,45)$ \\
Masculino & $0,84(0,76)$ & $0,72(0,60)$ & $0,75(0,64)$ & $0,74(0,62)$ & $0,56(0,35)$ & $0,55(0,34)$ \\
\hline \multicolumn{7}{c}{} \\
Total & $0,80(0,72)$ & $0,75(0,64)$ & $0,68(0,54)$ & $0,73(0,60)$ & $0,64(0,47)$ & $0,58(0,38)$ \\
\hline
\end{tabular}

Aunque no se preguntó la edad a los alumnos, esta se encontraba en torno a los 24 años. Los alumnos mostraron una alta implicación en la asignatura, realizando todas las tareas previstas y participando activamente en la asignatura. La asignatura está encuadrada en el segundo curso de un máster de Mejora Genética Vegetal, cuya aplicación práctica es muy evidente y el sector profesional donde los alumnos pueden emplearse está muy localizado. Por ello, la motivación de los alumnos suele ser muy alta y la aplicabilidad de los contenidos así lo favorece. Además, la metodología de la asignatua está enfocada a la discusión de conceptos y aplicaciones, lo que favorece su participación. Las metodologías activas favorecen el enfoque profundo y se recomienda su uso (Hall, Ramsay and Raven, 2004). Se ha sugerido que la carga excesiva de trabajo, determinados sistemas de evaluación, estilos de enseñanza o la baja proporción profesorado/estudiante fomentan el aprendizaje superficial (Gow, Kember and Cooper, 1994), mientras que el entusiamo de los profesores, la retroalimentación a los estudiantes o la generación de un ambiente personal de aprendizaje fomentan el aprendizaje profundo (Sharma, 1997). Cambios en el entorno de aprendizaje, tales como cambiar las actividades en el aula (Hall, Ramsay and Raven, 2004) o el uso de la clase inversa (Jeong et al., 2017), pueden tener efectos positivos sobre el enfoque de aprendizaje profundo del estudiante;. En nuestro caso, la metodología utilizada parece que fomenta el enfoque profundo, pero son necesarios más estudios para ver su influencia en el aprendizaje y en las calificaciones. También son necesarias más comparaciones con otras asignaturas del mismo y distinto contexto educativo para ver la importancia del contexto educativo concreto de la asignatura.

\section{Conclusiones}

El enfoque de aprendizaje de los estudiantes depende de factores personales y situacionales, pero se pueden clasificar en enfoque profundo y superficial. El entorno educativo y en particular la asignatura y el sistema de evaluación son factores claves en el enfoque de aprendizaje que opta el estudiante, ya que los estudiantes varían su enfoque en función de la asignatura y las expectavias que tienen. Los estudiantes 
participantes en el presente estudio mostraron un mayor enfoque de aprendizaje profundo que superficial, indicando una implición intrínseca en su aprendizaje que se manifiesta en su participación en la asignatura. No se detectó una influencia del genero del estudiante pero sí entre cursos. Las escalas principales del cuestionario mostraron altas correlaciones con las subescalas relacionadas y el cuestionario mostró una fiabilidad elevada para las escalas principales pero no para las secundarias. La metodología utilizada en la asignatura parece fomentar el enfoque profundo de aprendizaje, por lo que los esfuerzos de mejora en futuros cursos deben ir orientados en el mismo sentido. Sin embargo, se podrían implementar nuevas metodologías para mejorar el aprendizaje del alumno y fomentar aun más el enfoque profundo de los alumnos de la asignatura. Estudios de la variación del enfoque de aprendizaje al principio y final de la asignatura son necesarios para evaluar el efecto de la metodología de enseñanza.

\section{Agradecimientos}

La publicación de este trabajo ha sido parcialmente financiada por un proyecto de innovación educativa (PIME/2017/A/016/A y PIME/19-20/168) concedido por el Vicerrectorado de Estudios, Calidad y Acreditación de la Universitat Politècncia de València (UPV). También los autores agradecen el apoyo dado por el Instituto de Ciencias de la Educación (ICE) de la UPV.

\section{Referencias}

Biggs, J. (1987) Student Approaches to Learning and Studying. Research Monograph., Australian Education Research and Development. Available at: https://eric.ed.gov/?id=ED308201.

Biggs, J. B. and Tang, C. (2007) Teaching for quality learning at university. Berkshire: Open University Press/McGraw-Hill Education.

Biggs, J., Kember, D. and Leung, D. Y. P. (2001) 'The revised two-factor Study Process Questionnaire: R-SPQ-2F', British Journal of Educational Psychology, 71(1), pp. 133-149. doi: 10.1348/000709901158433.

Emilia, O., Bloomfield, L. and Rotem, A. (2012) 'Measuring students' approaches to learning in different clinical rotations', BMC Medical Education, 12(1), p. 114. doi: 10.1186/1472-6920-12-114.

Entwistle, N. and Entwistle, D. (2003) 'Preparing for examinations: The interplay of memorising and understanding, and the development of knowledge objects', Higher Education Research and Development, 22(1), pp. 19-41. doi: $10.1080 / 0729436032000056562$.

Entwistle, N. and Hilary, T. (2013) 'Approaches and Study Skills Inventory for Students (ASSIST) (incorporating the Revised Approaches to Studying Inventory - RASI)'. Available at: https://www.researchgate.net/publication/260291730_Approaches_and_Study_Skills_Inventory_for_Students_ASSI ST_incorporating_the_Revised_Approaches_to_Studying_Inventory_-_RASI.

Entwistle, N. J. and Tait, H. (1995) The revised approaches to studying inventory. Edinburgh, Scotland: Centre for Research on Learning and Instruction.

Entwistle, N., McCune, V. and Hounsell, J. (2002) Approaches to Studying and Perceptions of University TeachingLearning Environments: Concepts, Measures and Preliminary Findings, Report number: Occasional Report No 1 from ETL Project. Edinburgh. doi: 10.13140/RG.2.2.33594.80329.

Frăsineanu, E. S. (2013) 'Approach to Learning Process: Superficial Learning and Deep Learning at Students', Procedia - Social and Behavioral Sciences, 76, pp. 346-350. doi: 10.1016/j.sbspro.2013.04.125.

Fryer, L. K. et al. (2012) 'The adaptation and validation of the CEQ and the R-SPQ-2F to the Japanese tertiary environment', British Journal of Educational Psychology, 82(4), pp. 549-563. doi: 10.1111/j.20448279.2011.02045.x.

Gijbels, D. et al. (2005) 'The relationship between students' approaches to learning and the assessment of learning outcomes', European Journal of Psychology of Education, 20(4), pp. 327-341. doi: 10.1007/BF03173560. 
Gow, L. and Kember, D. (1990) 'Does higher education promote independent learning?', Higher Education, 19(3), pp. 307-322. doi: 10.1007/BF00133895.

Gow, L., Kember, D. and Cooper, B. (1994) 'The teaching context and approaches to study of accountancy students', Issues in Accounting Education, 9(1), pp. 118-130.

Hall, M., Ramsay, A. and Raven, J. (2004) 'Changing the learning environment to promote deep learning approaches in first-year accounting students', Accounting Education, 13(4), pp. 489-505. doi: 10.1080/0963928042000306837.

Hundleby, J. D. and Nunnally, J. (2006) Psychometric Theory, American Educational Research Journal. Edited by McGraw-Hil. New York, USA. doi: 10.2307/1161962.

Immekus, J. C. and Imbrie, P. K. (2010) 'A Test and Cross-Validation of the Revised Two-Factor Study Process Questionnaire Factor Structure Among Western University Students', Educational and Psychological Measurement, 70(3), pp. 495-510. doi: 10.1177/0013164409355685.

Jeong, J. S. et al. (2017) 'Examination of students ' engagement with R-SPQ- 2F of learning approach in flipped sustainable science course', pp. 880-891.

Justicia, F. et al. (2008) 'The Revised Two-Factor Study Process Questionnaire (R-SPQ-2F): Exploratory and confirmatory factor analyses at item level', European Journal of Psychology of Education, 23(3), pp. 355-372. doi: 10.1007/BF03173004.

Leiva-Brondo, M. et al. (2018a) 'DEEP LEARNING APPROACH FOR STUDENTS OF PLANT BREEDING IN A MASTER DEGREE', in Gómez Chova, L., López Martínez, A., and Candel Torres, I. (eds) 10th International Conference on Education and New Learning Technologies (EDULEARN18). Palma, Mallorca: International Association of Technology, Education and Development. IATED, pp. 3437-3442. doi: 10.21125/edulearn.2018.0890.

Leiva-Brondo, M. et al. (2018b) 'FIRST YEAR LIFE SCIENCE STUDENTS DEEP LEARNING APPROACH: A PRELIMINARY REPORT', in Gómez Chova, L., López Martínez, A., and Candel Torres, I. (eds) 11th International Conference of Education, Research and Innovation ICERI2018 Proceedings. Sevilla (Spain): International Association of Technology, Education and Development. IATED, pp. 542-548. doi: 10.21125/iceri.2018.1111.

Leiva-Brondo, M. et al. (2019) 'Evaluación de la aproximación al aprendizaje de estudiantes de ciencias de la vida usando la versión revisada del Study Process Questionnaire (R-SPQ-2F)', in Libro de Actas IN-RED 2019: V Congreso de Innovación Edicativa y Docencia en Red. València: Editorial Universitat Politècnica de València, pp. 156-170. doi: 10.4995/INRED2019.2019.10406.

Leung, D. Y. P., Ginns, P. and Kember, D. (2008) 'Examining the Cultural Specificity of Approaches To Learning in Universities in Hong Kong and Sydney', Journal of Cross-Cultural Psychology, 39(3), pp. 251-266. doi: $10.1177 / 0022022107313905$.

Marton, F. and Säljö, R. (1976a) 'On qualitative differences in learning-II outcome as a function of the learners's conception of the task', British Journal of Educational Psychology, 46(2), pp. 115-127. doi: 10.1111/j.20448279.1976.tb02304.x.

Marton, F. and Säljö, R. (1976b) 'On qualitative differences in learning: I-Outcome and process', British Journal of Educational Psychology, 46(1), pp. 4-11. doi: 10.1111/j.2044-8279.1976.tb02980.x.

Michael, W. B., Michael, J. J. and Zimmerman, W. S. (1985) Study Attitudes and Methods Survey (SAMS). San Diego, CA: Educational and Industrial Testing Service.

Mogre, V. and Amalba, A. (2014) 'Assessing the reliability and validity of the Revised Two Factor Study Process Questionnaire (RSPQ2F) in Ghanaian medical students', Journal of Educational Evaluation for Health Professions, 11, p. 19. doi: 10.3352/jeehp.2014.11.19.

Muñoz San Roque, I., Prieto Navarro, L. and Torre Puente, J. C. (2012) 'Enfoques de aprendizaje, autorregulación, autoeficacia, competencias y evaluación. Un estudio descriptivo de estudiantes de educación infantil y primaria', in Torre Puente, J. C. (ed.) Educación y nuevas sociedades. Madrid: Universidad Pontificia Comillas, pp. $237-266$.

Richardson, J. T. E. (2013) 'Approaches to studying across the adult life span: Evidence from distance education', Learning and Individual Differences. Elsevier Inc., 26, pp. 74-80. doi: 10.1016/j.lindif.2013.04.012.

Richardson, J. T. E. and King, E. (1998) 'Adult Students in Higher Education: Burden or Boon?', The Journal of Higher Education, 69(1), p. 65. doi: 10.2307/2649182.

Rubin, M. et al. (2018) 'Older Women, Deeper Learning, and Greater Satisfaction at University: Age and Gender Predict University Students' Learning Approach and Degree Satisfaction', Journal of Diversity in Higher Education, 11(1), pp. 82-96. doi: 10.1037/dhe0000042. 
Salamonson, Y. et al. (2013) 'Learning approaches as predictors of academic performance in first year health and science students', Nurse Education Today. Elsevier Ltd, 33(7), pp. 729-733. doi: 10.1016/j.nedt.2013.01.013.

Schmeck, R. R., Ribich, F. and Ramanaiah, N. (1977) 'Development of a Self-Report Inventory for Assessing Individual Differences in Learning Processes', Applied Psychological Measurement, 1(3), pp. 413-431. doi: $10.1177 / 014662167700100310$.

Shaik, S. A. et al. (2017) 'Assessing Saudi medical students learning approach using the revised two-factor study process questionnaire', International Journal of Medical Education, 8, pp. 292-296. doi: 10.5116/ijme.5974.7a06.

Sharma, D. S. (1997) 'Accounting students' learning conceptions, approaches to learning, and the influence of the learning-teaching context on approaches to learning', International Journal of Phytoremediation, 21(1), pp. $125-146$. doi: 10.1080/096392897331532.

Socha, A. and Sigler, E. A. (2014) 'Exploring and "reconciling" the factor structure for the Revised Two-factor Study Process Questionnaire', Learning and Individual Differences. Elsevier Inc., 31, pp. 43-50. doi: 10.1016/j.lindif.2013.12.010.

Stes, A., de Maeyer, S. and Van Petegem, P. (2013) 'Examining the Cross-Cultural Sensitivity of the Revised TwoFactor Study Process Questionnaire (R-SPQ-2F) and Validation of a Dutch Version', PLoS ONE. Edited by O. García, 8(1), p. e54099. doi: 10.1371/journal.pone.0054099.

Swee Choo Goh, P. (2006) 'Assessing the Approaches to Learning of Twinning Programme Students in Malaysia', Malaysian Journal of Learning and Instruction, 3(1), pp. 93-115.

Tait, H., Entwistle, N. J. and McCune, V. (1998) 'ASSIST: a reconceptualisation of the Approaches to Studying Inventory', in Rust, C. (ed.) Improving students as learners. Oxford: Oxford Brookes University, The Oxford Centre for Staff and Learning Development., pp. 262-271.

Vermunt, J. D. (1994) Inventory of Learning Styles in Higher Education: Scoring key. Tilburg, The Netherlands: Tilburg University, Department of Educational Psychology.

Weinstein, C. E. (1987) Learning and Study Strategies Inventory. Clearwater, FL: H \& H Publishing.

Zakariya, Y. F. (2019) 'Study approaches in higher education mathematics: Investigating the statistical behaviour of an instrument translated into norwegian', Education Sciences, 9(3). doi: 10.3390/educsci9030191.

Zeegers, P. (2001) 'Approaches to learning in science: A longitudinal study', British Journal of Educational Psychology, 71(1), pp. 115-132. doi: 10.1348/000709901158424. 